\title{
PATH OPTIMIZATION ALGORITHM FOR NETWORK PROBLEMS USING JOB SEQUENCING TECHNIQUE
}

\author{
Punit Kumar Singh $^{1}$ and Dr. Rakesh Kumar ${ }^{2}$ \\ ${ }^{1}$ Department of Computer Science and Engineering, M.M.M Engineering College, \\ Gorakhpur-273010, India \\ punit20.singh@gmail.com \\ ${ }^{2}$ Department of Computer Science and Engineering, M.M.M Engineering College, \\ Gorakhpur-273010, India \\ rkiitregmail.com
}

\section{ABSTRACT:}

The job sequencing technique is used to determine an optimal sequence. It performs a series of jobs by a number of specific orders so that it calculates the optimal cost. In this paper, we propose a novel approach to find an optimal path from source to destination by taking advantage of job sequencing technique. We have used $n$ jobs $m$ machine sequencing technique and this is divided into $n$ jobs 2 machine problems. Using Johnson's sequencing rule, we solved the problem and obtained the (n-1) sub sequences of the route. Using the proposed algorithm, we calculated the optimal sequence, which leads to the shortest path of the network.

\section{KEYWORD :}

Job sequencing technique, Optimal sequence, minimal path, optimal distance, Johnson's sequencing rule, MPLS. .

\section{INTRODUCTION}

Routing problems in networks are the problem related to sequencing and, of late, they have been receiving increasing attention. Such problems usually occur in the areas of transportations and communication. A network problem involves the determination of a route from source city I to destination city $\mathrm{J}$ for there exist a number of alternative paths at various stages of the journey. The cost of journey, which may be function of distance, time or money, is different for different routes and the problem is to find the minimum cost route. Theoretically, the procedure involves determination of the cost of all the possible routes and selecting the one with minimum cost. However, in actual practice the number of such feasible alternatives is too large to be tried one by one. The travelling salesman problem is a routing problem involving rather severe constraints. Another routing problem arises when we wish to go from one place to another or to several other places and we are to select the shortest route involving least distance or time or cost out of many alternatives, to reach the desired station. Such acyclic route network problem can be easily solved by job sequencing technique. A network is defined as a set of points or nodes which are connected by lines or links. A way of going from one node to another is called a route or path. The problem of sequencing may have some restrictions placed on it, such as time for each job on each machine, availability of resources (men, machine, material, and space) etc. in sequencing problem, the effectiveness can be measured in terms of minimized cost, maximized profits, and minimized elapsed times. But in this paper we construct $M \times M$ adjacency matrices between nodes and corresponding weight of the edges. Here $\mathrm{M}$ denotes the node of the network. Instead of time for each job for each machine we required the distance from one node to another node. And calculate the optimal sequence which occur minimal total distance between source to destination. 
J. Salcedo Parra showed that optimal routing [13] solutions may be found for a diverse set of traffic engineering goals. However, for a network with $\mathrm{N}$ nodes (routers) and $\mathrm{M}$ edges (links), their MPLS implementation may use $(\mathrm{N} \times \mathrm{M})$ [11] different labels.

The rest of the paper is organized as follows: In section 2, we mention related works carried out by other researchers. Proposed algorithm to find the shortest path of a given complex network is presented in section 3. In section 4, we present our results and its analysis. Finally, conclusion and future scope have been given under section 5 .

\section{RELATED WORK}

Many research articles [5, 6, 7, 8, 9] deal for framing optimal job sequences in job sequencing technique under various circumstances. The paper [1] studies the single job streaming problem in a two stage hybrid flow shop that has $\mathrm{n}$ identical machine at the first stage and single machine at the second stage to minimize the span and the article [3] consider the one machine bicriterion scheduling problem of enumerating pare to optimal sequences with respect to the total weight, completion time and the maximum lateness objectives. The paper [2] deals with the problem of minimizing the make span in a two machine flow shop. When the job processing time is deterministic, the optimal job sequence technique can be determined by applying Johnson's rule. When they are independent and exponential random variables, Talwar's rule yields a job sequence that minimizes the make distance stochastically. Assuming that the job processing times are separately and Weibull distributed random variables a novel job sequencing rule is presented that includes equally Johnson's and Talwar's rules as special cases. The paper [3] addresses a problem of continuous batch scheduling arising in the heating process of blooms in steel diligence and the editorial [4] presented a simple, elegant algorithm for finding an optimal solution to a general min - max sequencing problem. In the editorial [8] the problem of sequencing $n$ jobs in a two machine reentrant shop with the objectives of minimizing the maximum completion time is considered. The editorial [9] is concerned with the problem of scheduling $\mathrm{n}$ jobs with a general due date on a single machine so as to diminish the total outlay arising from earliness and lateness and the editorial [10] gives computer program for solving $\mathrm{m}$ jobs $\mathrm{n}$ machine sequencing problems with the help of 2 jobs $n$ machine sequencing problems and this technique is used in this paper to find shortest path between 2 nodes in complex networks.

\section{PROPOSED WORK}

The following algorithm is used to find the shortest path of the given complex network. For this, it generates up to $\mathrm{n}-1$ sequences. Sequence generation is accomplished in the following manner: Let $t_{j i}$ where $j=1,2,3, \ldots \ldots \ldots, n$ and $i=1,2,3, \ldots \ldots \ldots \ldots ., n$ represent the distance having $j^{\text {th }}$ node from the $\mathrm{i}^{\text {th }}$ node. In this algorithm firstly we make a weighted adjacency matrix using the given network having node and edge having some weight which denotes distance between the two nodes. Now we have divided it ( $\mathrm{N}$ x N weighted matrix) into N X 2 sub matrix according to the given formula.

$\mathrm{M}_{\mathrm{j} 1}{ }^{\mathrm{k}}=\sum_{\mathrm{i}=1}^{\mathrm{k}} \mathrm{t}_{\mathrm{ji}}=$ Constructing first column of $\mathrm{N} \times 2$ adjacency matrices.

$\mathrm{M}_{\mathrm{j} 2}{ }^{\mathrm{k}}=\sum_{\mathrm{i}=\mathrm{n}+1-\mathrm{k}}^{\mathrm{n}} \mathrm{t}_{\mathrm{ji}}=$ Constructing second column of $\mathrm{N} \times 2$ adjacency matrices.

After it using Johnson's rule we have sequence the node so that we have obtain n-1 sequences. Then we calculate the cost of each and every sub sequence. Now estimate which sequence cost is minimum that is optimal sequence and provides shortest path of the network. This algorithm follows the following step. 
Step 1: Begin

Step 2: Construct the $\mathrm{N} x \mathrm{~N}$ adjacency matrices Where $\mathrm{N}$ is the node of the network.

Step 3: The $\mathrm{N} x \mathrm{~N}$ adjacency matrices split into $\mathrm{N} \times 2$ sub matrices. The number of such matrices will $\mathrm{b} N-1$. Thus a network having 7 nodes then it will involve 7-1=6 sub matrices.

Step 4: Using $\mathrm{p}$, where $\mathrm{p}<=\mathrm{N}-1$, auxiliary N-1 sub matrices can be defined as follows. In the $\mathrm{K}^{\text {th }}$ auxiliary problem.

Step 5: Set k=1, for first auxiliary problem.

$\mathrm{M}_{\mathrm{j} 1}{ }^{\mathrm{k}}=\sum_{\mathrm{i}=1}^{\mathrm{k}} \mathrm{t}_{\mathrm{ji}}=$ Constructing first column of $\mathrm{N} \times 2$ adjacency matrices.

$\mathrm{M}_{\mathrm{j} 2}{ }^{\mathrm{k}}=\sum_{\mathrm{i}=\mathrm{n}+1-\mathrm{k}}^{\mathrm{n}} \mathrm{t}_{\mathrm{ji}}=$ Constructing second column of $\mathrm{N} \times 2$ adjacency matrices.

Step 6: Apply S.M. Johnson's n-job, 2- machine algorithm to the n-job 2-machine problems established and determine $S_{k}$ and store.

Step 7: Check $k$ with $p$; if $k<p$, set $k=k+1$ and repeat the step 4; if $k=p$, then proceed.

Step 8: Using real $\mathrm{N} x \mathrm{~N}$ matrix of processing distance, compute total processing distance for each of the $\mathrm{p}$ sequences generated.

Step 9: Select minimum total processing distance sequence as the optimal sequence. This optimal sequence is determining the shortest path of the given complex network.

Step 10: End.

\section{RESULT AND ANALYSIS}

Consider the Fig 1 which is a network having 5 nodes and corresponding weight of the edge is labeled as in the figure. The weight represent the distance between one node from the another node. Now we have to determine the shortest path between source nodes to the destination node using the above given algorithm or proposed model. We have simulated this algorithm in language ' $C$ ' and according to the simulation result we have find the $n-1$ sequences and each sequence having some execution cost which sequence having minimum cost which is optimal path. Examples are given bellows. 
International Journal of Distributed and Parallel Systems (IJDPS) Vol.3, No.3, May 2012

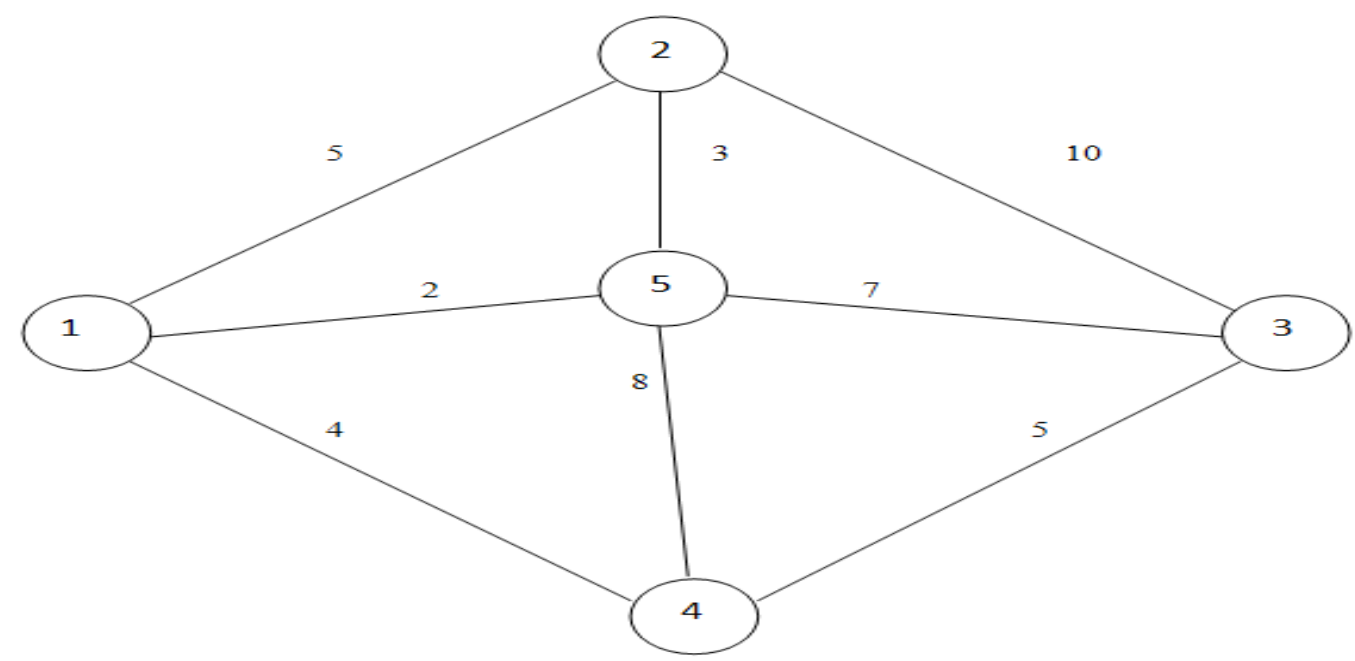

Figure 1: weighted network

\subsection{ADJACENCY MATRIX}

\begin{tabular}{|c|c|c|c|c|c|}
\hline NODE & 1 & 2 & 3 & 4 & 5 \\
\hline 1 & 0 & 5 & 0 & 4 & 2 \\
\hline 2 & 5 & 0 & 10 & 0 & 3 \\
\hline 3 & 0 & 10 & 0 & 5 & 7 \\
\hline 4 & 4 & 0 & 5 & 0 & 8 \\
\hline 5 & 2 & 3 & 7 & 8 & 0 \\
\hline
\end{tabular}

\subsection{SUB MATRIX1}

\begin{tabular}{|c|c|c|}
\hline NODE & N1 & N5 \\
\hline 1 & 0 & 2 \\
\hline 2 & 5 & 3 \\
\hline 3 & 0 & 7 \\
\hline 4 & 4 & 8 \\
\hline 5 & 2 & 0 \\
\hline
\end{tabular}




\subsection{SUB MATRIX2}

\begin{tabular}{|c|c|c|}
\hline NODE & N1 + N2 & N4 + N5 \\
\hline 1 & 5 & 6 \\
\hline 2 & 5 & 3 \\
\hline 3 & 10 & 8 \\
\hline 4 & 5 & 8 \\
\hline 5 & & 12 \\
\hline
\end{tabular}

\subsection{SUB MATRIX3}

\begin{tabular}{|c|c|c|}
\hline NODE & N1 + N2 + N3 & N3 + N4 + N5 \\
\hline 1 & 5 & 6 \\
\hline 2 & 15 & 12 \\
\hline 3 & 10 & 13 \\
\hline 4 & 9 & 15 \\
\hline
\end{tabular}

\subsection{SUB MATRIX4}

\begin{tabular}{|c|c|c|}
\hline NODE & N1 + N2 + N3 +N4 & N2 + N3 + N4 + N5 \\
\hline 1 & 9 & 11 \\
\hline 2 & 15 & 13 \\
\hline 3 & 15 & 13 \\
\hline 4 & 9 & 18 \\
\hline 5 & 20 & \\
\hline
\end{tabular}


International Journal of Distributed and Parallel Systems (IJDPS) Vol.3, No.3, May 2012

4.6. OPTIMAL SEQUENCE OF EACH SUB MATRIX

SEQUENCE OF SUB MATRIX 1

\begin{tabular}{|c|c|c|c|c|c|}
\hline SEQUENCE/NODE & $\mathbf{1}$ & $\mathbf{2}$ & $\mathbf{3}$ & $\mathbf{4}$ & $\mathbf{5}$ \\
\hline $\mathbf{1}$ & $\mathbf{0}$ & $\mathbf{5}$ & $\mathbf{5}$ & $\mathbf{9}$ & $\mathbf{1 1}$ \\
\hline 3 & 0 & 15 & 15 & 20 & 27 \\
\hline 4 & 4 & 15 & 20 & 20 & 35 \\
\hline 2 & 9 & 15 & 30 & 30 & 38 \\
\hline 5 & 11 & 18 & 37 & 45 & 45 \\
\hline
\end{tabular}

SEQUENCE OF SUB MATRIX 2

\begin{tabular}{|c|c|c|c|c|c|}
\hline NODE NO. & $\mathbf{1}$ & $\mathbf{3}$ & $\mathbf{4}$ & $\mathbf{2}$ & $\mathbf{5}$ \\
\hline
\end{tabular}

SEQUENCE OF SUB MATRIX 3

\begin{tabular}{|l|l|l|l|l|l|}
\hline NODE NO. & $\mathbf{4}$ & $\mathbf{1}$ & $\mathbf{5}$ & $\mathbf{3}$ & $\mathbf{2}$ \\
\hline
\end{tabular}

SEQUENCE OF SUB MATRIX

\begin{tabular}{|l|l|l|l|l|l|}
\hline NODE NO. & 1 & 4 & 3 & 5 & 2 \\
\hline
\end{tabular}

\begin{tabular}{|l|l|l|l|l|l|}
\hline NODE NO. & $\mathbf{1}$ & $\mathbf{4}$ & $\mathbf{3}$ & $\mathbf{5}$ & $\mathbf{2}$ \\
\hline
\end{tabular}

4.7. TOTAL PROCESSING DISTANCE IS CALCULATED FOR EACH DIFFERENT SEQUENCE

For Sequence $\quad 1 \rightarrow 3 \rightarrow 4 \rightarrow 2 \rightarrow 5$

Total distance $=45$

For Sequence $4 \rightarrow 1 \rightarrow 5 \rightarrow 3 \rightarrow 2$

Total distance $=42$

\begin{tabular}{|c|c|c|c|c|c|}
\hline SEQUENCE/NODE & 1 & 2 & 3 & 4 & 5 \\
\hline 4 & 4 & 4 & 9 & 9 & 17 \\
\hline 1 & 4 & 9 & 9 & 13 & 29 \\
\hline 5 & 6 & 12 & 19 & 27 & 27 \\
\hline 3 & 6 & 22 & 22 & 32 & 39 \\
\hline 2 & 11 & 22 & 32 & 32 & 42 \\
\hline
\end{tabular}


For Sequence $\quad 1 \rightarrow 4 \rightarrow 3 \rightarrow 5 \rightarrow 2$

Total distance $=37$

\begin{tabular}{|c|c|c|c|c|c|}
\hline SEQUENCE/NODE & $\mathbf{1}$ & $\mathbf{2}$ & $\mathbf{3}$ & $\mathbf{4}$ & $\mathbf{5}$ \\
\hline $\mathbf{1}$ & $\mathbf{0}$ & $\mathbf{5}$ & $\mathbf{5}$ & $\mathbf{9}$ & $\mathbf{1 1}$ \\
\hline $\mathbf{4}$ & $\mathbf{4}$ & $\mathbf{5}$ & $\mathbf{1 0}$ & $\mathbf{1 0}$ & $\mathbf{1 9}$ \\
\hline 3 & 4 & 15 & 15 & 20 & 27 \\
\hline $\mathbf{5}$ & $\mathbf{6}$ & 18 & 22 & 30 & 30 \\
\hline $\mathbf{2}$ & 11 & 18 & 32 & 32 & 37 \\
\hline
\end{tabular}

In the above mathematical calculation minimum distance 35 is obtained which follows the sequence $1 \rightarrow 4 \rightarrow 3 \rightarrow 5 \rightarrow 2$. Hence the optimal route or path of the network is $1 \rightarrow 4 \rightarrow 3 \rightarrow 5 \rightarrow 2$ which is shown in Fig 2.

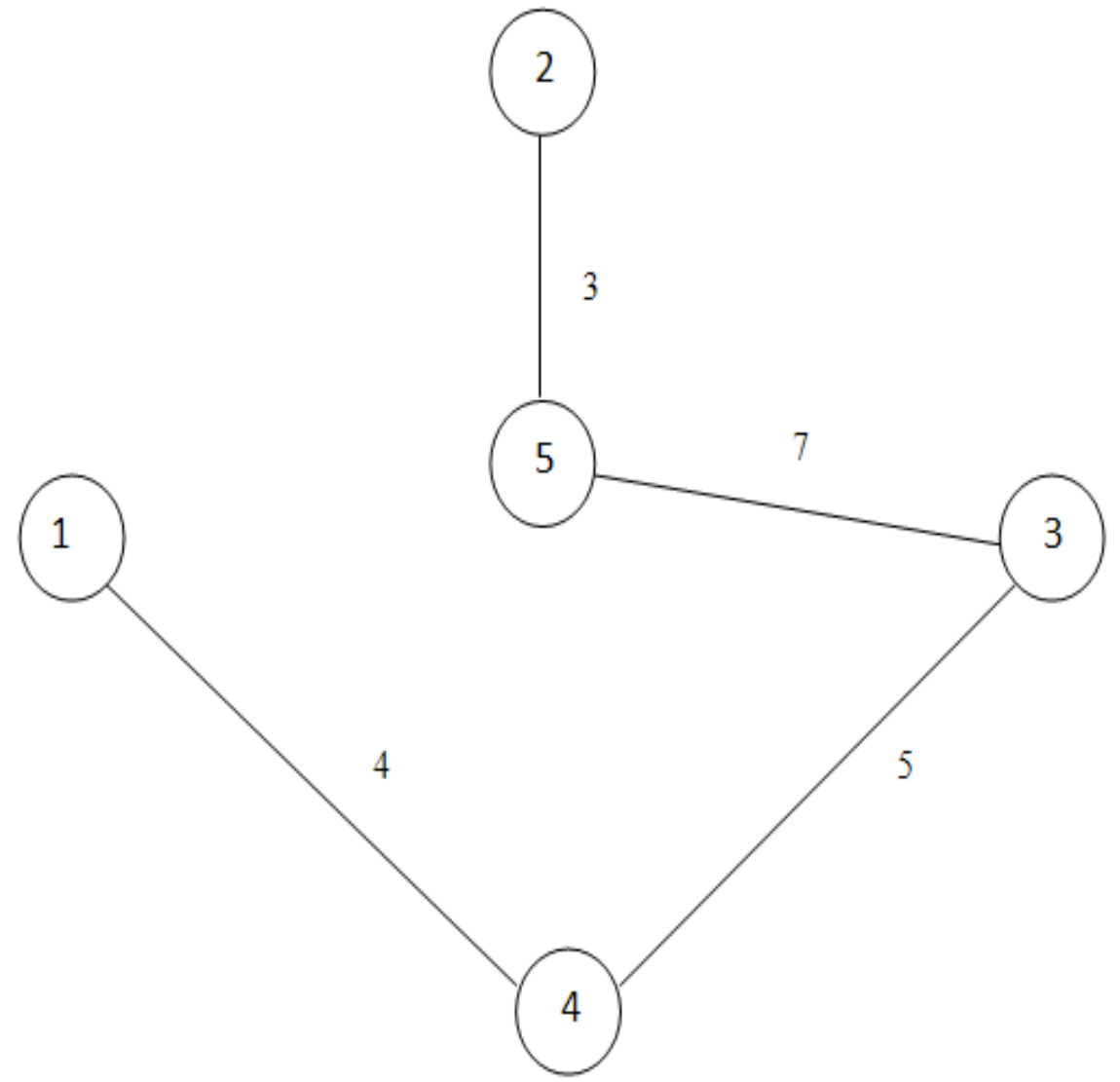

Figure 2: Optimal path 


\section{CONCLUSIONS AND FUTURE SCOPE}

In this paper, we have to find the optimized path of the network problems using $\mathrm{N} \times \mathrm{M}$ sequencing problems by solving $\mathrm{N} x 2$ job sequencing technique. The $\mathrm{n}$ jobs $\mathrm{m}$ machine problems or technique only used for machine means hardware for sequencing the jobs but in this paper this technique is used in networking problem like as shortest route finding problem. Here we consider $\mathrm{N} \times \mathrm{N}$ weighted adjacency matrix which includes node vs. node where weight means distance between two node means edge between the nodes for this we consider the graph in figure 1 and find out the shortest path shown in figure2. This is suitable for hardware and software both. And in near future this algorithm is extended in new heuristic method to determine an optimal searching technique without using existing conditions. And now we are using this algorithm in multi-protocol label switching [12] for sequencing the label. And route optimization.

\section{REFERENCES}

[1] Christos Koulamas, George J.kyparisis, "Single machine and two machine flow shop scheduling with general functions", European Journal of Operation Research Vol. 178, pp. 402-407, 2007.

[2] Kalczynski.P.J, Kamburowski.J., "A heuristic for minimizing the expected makespan in two machine flow shops with consistent coffiecient of variation", European Journal of Operation Research Vol. 169, pp. 742-750, 2006.

[3] Zhao.Y.F., Tang. L.X., "Scheduling a single continuous batch processing machine to minimize total completion time", Tien Tzu Hsuch pao/Acta Electronica Sinica, Vol. 36 pp. 367-370,2008

[4] Pan.Y.,Shi.L., Zhang.H, “ A new solution approach to the general min-max sequencing problem”, proceedings of the World Class on Intelligent Control and Automation(WCICA), Vol.1, pp.13591364,2006 .

[5] Corlier. J., Pinston. E., "A practical use of Jackson's preemptive scheduling for solving the job shop problem", Annals of Operation Research, Vol. 26, pp. 269-287,1990.

[6] Elmaghraby, S.E, Thoney.K.A., "The two machine stochastic flow shop problem with arbitrary processing time distributions", IIE Transactions, Vol. 31, pp. 467-477,1999.

[7] Cai. X.,Zhou. S., " Sequencing jobs with random processing time variance", Annals of Operation Research, Vol. 70, pp. 241-260,1997.

[8] Levin.V.I., Miretskii, I.Yu, "Optimal job scheduleing for flow shop", Automation and Remark Control, Vol.57, pp. 773-793, 1996.

[9] Srinivas.J., Subbiah.K.V., Chandramouli K.V.V., "An optimal sequencing approach for job shop production", Journal of Scientific and Industrial Research", Vol.63, pp. 458-461, 2004.

[10] Karthikeyan K., "Heuristic algorithm for job sequencing problems", Global Journal of Science Frontier Research, Vol.10 Isuue 4(Ver 1.0), September 2010.

[11] David Applegate "Load optimal MPLS routing with N + M labels", vol.0-7803,pp.7753-2/03, IEEE INFOCOM 2003.

[12] Rolf Winter, "Rolf Winter, NEC Labs Europe",NEC Labs Europe, vol. 0163-6408/11, IEEE Communications Magazine, April 2011.

[13] J. Salcedo Parra," Dijkstra's Algorithm Model Over MPLS / GMPLS”, vol. 978-1-4244-62520/11/2011 IEEE. 


\section{Authors.}

Mr. Punit Kumar Singh has received his B. Tech. in Computer Science and Engineering from IIMT Engineering College, Meerut (UP) Presently, he is pursuing his M .Tech program from the department of Computer Science and Engineering at M.M.M. Engineering College Gorakhpur India. His research interest is in Computer Networks.

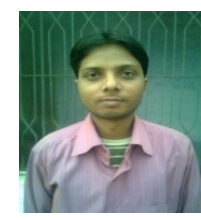

Dr. Rakesh Kumar received $\mathrm{PhD}$ in Computer Science \& Engineering from Indian Institute of Technology Roorkee, India in 2011, M.E. in Computer Engineering from S.G.S. Institute of Technology and Science Indore, India in 1994 and B. E. in Computer Engineering (First class with Hons.) from M. M. M. Engineering College, Gorakhpur, UP, India in 1990. Dr. Kumar is in teaching, research \& development since 1992 and is presently working as an Associate Professor in the Department of Computer Science and Engineering, M.M.M. Engineering College Gorakhpur-India. He has published many research papers in many refereed International/National

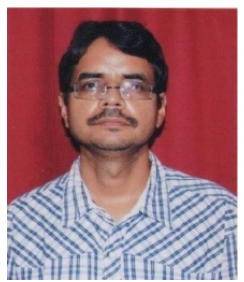
Journals and International Conferences. He had been awarded for Best Research paper in an International conference. He is Fellow of IETE and IE and also member of CSI and ISTE. His research interests are in Mobile \& Distributed Computing, Mobile Ad hoc Routing, Quality of Service Provisioning, Sensor Networks and Performance Evaluation. 